\title{
Relações solo-vegetação na estruturação de comunidades de cerrado sensu stricto no sul de Minas Gerais, Brasil
}

Soil-vegetation relationships in structuring cerrado sensu stricto communities in southern Minas Gerais, Brazil

\author{
Daniel Mendonça Torres ${ }^{1,3}$, Marco Aurélio Leite Fontes ${ }^{1}$ \& Henrique do Prado Samsonas ${ }^{2}$
}

\begin{abstract}
Resumo
Os cerrados do sul de Minas Gerais ainda são pouco estudados. Objetivou-se com esse trabalho compreender o papel dos solos na estruturação de duas comunidades arbóreas de cerrado no município de Guapé. Para isso, foram instaladas 14 parcelas de $50 \times 30 \mathrm{~m}$ em dois fragmentos de cerrado sendo 8 sobre Neossolo Litólico e 6 sobre Argissolo Vermelho. Nessas parcelas, todos os indivíduos arbóreos com circunferência a $30 \mathrm{~cm}$ do solo $(\mathrm{C} 30) \geq 15,7 \mathrm{~cm}$ (diâmetro de $5 \mathrm{~cm}$ ) foram medidos e tiraram-se amostras de solo de cada parcela para análises químicas e texturais. Ao todo foram amostrados 1561 indivíduos e a riqueza florística total foi de 83 espécies pertencentes a 65 gêneros de 37 famílias. A área sobre Neossolo foi classificada como cerrado sensu stricto rupestre e Caryocar brasiliense foi a espécie mais importante em VI para a fisionomia. O cerrado sobre Argissolo foi categorizado como um cerrado sensu stricto típico e Dalbergia miscolobium foi a espécie de maior VI para essa fitofisionomia. A análise de correspondência canônica apresentou autovalor significativo para o primeiro eixo de 0,563 indicando gradiente curto com rápida substituição de espécies. Encontrou-se associação aos teores de $\mathrm{P}$ e areia no cerrado rupestre e de $\mathrm{K}$ e argila no cerrado típico. Por fim, a distribuição das espécies no gradiente foi diferente entre os substratos indicando preferência de espécies por sítios específicos. A conservação dos cerrados do sul de Minas Gerais deve, portanto, considerar não apenas a diversidade florística, mas suas múltiplas pedoformas e as formações ecotonais associadas.
\end{abstract}

Palavras-chave: florística, fitogeografia, hotspot, savana.

\begin{abstract}
The cerrados of southern Minas Gerais are still poorly studied. The objective of this work was to understand the role of soils in structuring two tree communities of cerrado in the municipality of Guapé. For that 14 plots of $50 \times 30 \mathrm{~m}$ were installed in two cerrado fragments being 8 over Litholic Neosol and 6 over Red Argisol. In these plots, all tree individuals with circumference at $30 \mathrm{~cm}$ from the ground $(\mathrm{C} 30) \geq 15.7 \mathrm{~cm}$ ( $5 \mathrm{~cm}$ diameter) were measured and soil samples from each plot were taken for chemical and textural analysis. In total we sampled 1561 individuals and total species richness was 83 species belonging to 65 genera of 37 families. The area on Neossol was classified as rupestral cerrado sensu stricto and Caryocar brasiliense was the most important species in VI in the physiognomy. The cerrado on Argisol was categorized as typical cerrado sensu stricto and Dalbergia miscolobium was the largest species in VI to this vegetation type. The CCA presented a significant eigenvalue to the first axis of 0.563 indicating a short gradient with rapid replacement of species. There was an association at the contents of $\mathrm{P}$ and sand in the rupestral cerrado and $\mathrm{K}$ and clay in the typical cerrado. Finally, the distribution of species in the gradient was different between the substrates indicating species preference for specific sites. The conservation of the cerrados of southern Minas Gerais should therefore consider not just the floristic diversity but their multiple landforms and associated ecotones formations.

Key words: floristics, phytogeography, hotspot, savannah.
\end{abstract}

\footnotetext{
${ }^{1}$ Universidade Federal de Lavras, Depto. Ciências Florestais, Campus Universitário, C.P. 3037, 37200-000, Lavras, MG, Brasil.

${ }^{2}$ Universidade Federal Fronteira Sul, Depto. Agronomia, Av. Fernando Machado 108E, 89802-112, Chapecó, SC, Brasil.

${ }^{3}$ Autor para correspondência: daniel_bioagro@yahoo.com.br
} 


\section{Introdução}

O Domínio Fitogeográfico do Cerrado abrange diversos tipos de vegetação, com amplas variações na composição de espécies e na estrutura entre eles (Ribeiro \& Walter 2008). Majoritariamente coberto pelo Cerrado sensu stricto (Eiten 1994), os fatores que determinam as variações dessa fisionomia em regiões ecótones, no entanto ainda não estão suficientemente claros (Durigan \& Ratter 2006; Dantas \& Batalha 2010). Diversos trabalhos correlacionam a riqueza e a biomassa de comunidades arbóreas de cerrados com gradientes de fertilidade do solo (Miranda et al. 2002; Oliveira-Filho \& Ratter 2002; Goodland \& Pollard 1973). Há estudos que justificam a limitação da distribuição de espécies de acordo com o nível de alumínio no substrato (Carvalho \& Martins 2009). Já outros autores associam a distribuição diferencial das espécies em função da disponibilidade de água no solo (Furley \& Ratter 1988; Assis et al. 2011).

De modo similar à pluralidade de fitofisionomias, o Domínio do Cerrado abrange diversas geomorfologias e pedoformas (Ab'Sáber 2003). Ao longo de seus $2.000 .000 \mathrm{~km}^{2} \mathrm{de}$ extensão, os Latossolos predominam com $40 \%$ do total, enquanto os Neossolos Litólicos correspondem a 7,3\% e os Argissolos a 15,1\% (Reatto et al. 1998). As formações fitofisonômicas predominantes sobre estas classes de solo são respectivamente os cerrados sensu stricto típicos nos Latossolos e Argissolos, e os cerrados sensu stricto rupestres sobre Neossolos (Reatto et al. 1998). Embora seja uma das vegetações mais bem estudadas do Brasil, as áreas de transição entre o Cerrado e a Mata Atlântica (e.g., Florestas Estacionais Semideciduas - FESD) são exemplos de regiões que receberam pouca atenção (Durigan \& Ratter 2006), e a fitossociologia tem sido um recurso de grande valia para aumentar o conhecimento da flora dos Cerrados e destacar as diferenças entre as fitofisionomias do Domínio (Ribeiro \& Walter 2008).

Em Minas Gerais, Moreno \& Schiavini (2001), concluíram que os fatores edáficos desempenham um papel fundamental na estrutura e composição florística ao longo de gradientes de floresta para o cerrado. Em São Paulo, Durigan et al. (2003), afirmam que a gradual transição entre os cerrados e as florestas semidecíduas atlânticas ocorre ao longo de um gradiente de fertilidade e capacidade de retenção de água. No sul de Minas Gerais, poucos trabalhos foram desenvolvidos em cerrados (Carvalho \& Martins 2009; Carvalho \& Martins 1994), e das duas áreas abordadas por estes, nenhum foi desenvolvido em cerrados rupestres. Por outro lado, embora seja considerável o número de trabalhos com cerrados rupestres no Brasil (Amaral et al. 2006; Moura et al. 2007, 2010; Pinto et al. 2009), não há registros de levantamentos fitossociológicos de Cerrados sensu stricto sobre Argissolos, apenas de formações florestais (Borem \& Oliveira-Filho 2002; Carvalho et al. 2007).

Segundo Ab'Sáber (2003), em parte da história de ocupação do cerrado, latifúndios de pecuária de baixo nível de aperfeiçoamento permitiram a manutenção de relictos de vegetação mais bem conservada. Este é o caso da maioria dos cerrados do sul de Minas Gerais. Porém, ainda segundo Ab'Sáber (2003), sob essas condições históricas, tais zonas relictuais de cerrado devem ser melhor atendidas com investimentos múltiplos tornando-se, dessa forma, reservas especiais para o futuro. Neste trabalho, portanto, objetivou-se compreender o papel dos solos na ecologia e estruturação dos cerrados sensu stricto através do levantamento florístico e fitossociológico de duas comunidades arbóreas no sul de Minas Gerais, bem como realizar suas caracterizações fitofisionômicas e pedológicas.

\section{Material e Métodos}

Área de estudo

O presente estudo, localizado pela Fig. 1, foi desenvolvido no município de Guapé, Sul do estado de Minas Gerais, na bacia do médio rio Grande, às margens do Reservatório de Furnas. O clima local é do tipo Cwb de Köppen, ou seja, mesotérmico com invernos secos e verões brandos e chuvosos. Com altitudes variando de 788 a $932 \mathrm{~m}$, o índice médio pluviométrico anual é de $1.448 \mathrm{~mm}$ e a temperatura média de $21,2^{\circ} \mathrm{C}$ (Brasil 1962). O primeiro fragmento estudado ( $138,3 \mathrm{ha})$ foi classificado segundo Ribeiro \& Walter (2008), como um cerrado sensu stricto subtipo rupestre, situado nas coordenadas geográficas 20 49 '13.80"S e $46^{\circ} 00^{\prime} 39.41^{\prime \prime}$ O. Enquanto o segundo (71,6 ha) um cerrado sensu stricto subtipo típico localizado pelas coordenadas $20^{\circ} 51^{\prime} 20.92$ 'S e 46 2'12.90"O. A paisagem da região é aquela marcada pelo relevo moderadamente ondulado a acentuado. Nos fundos de vales e beiras de cursos de água encontram-se as matas de galeria, similarmente às descrições de Ab'Sáber (2003) para a área core do Domínio do Cerrado. Nas porções médias e altas do relevo encontram-se os mosaicos de vegetação típicos 


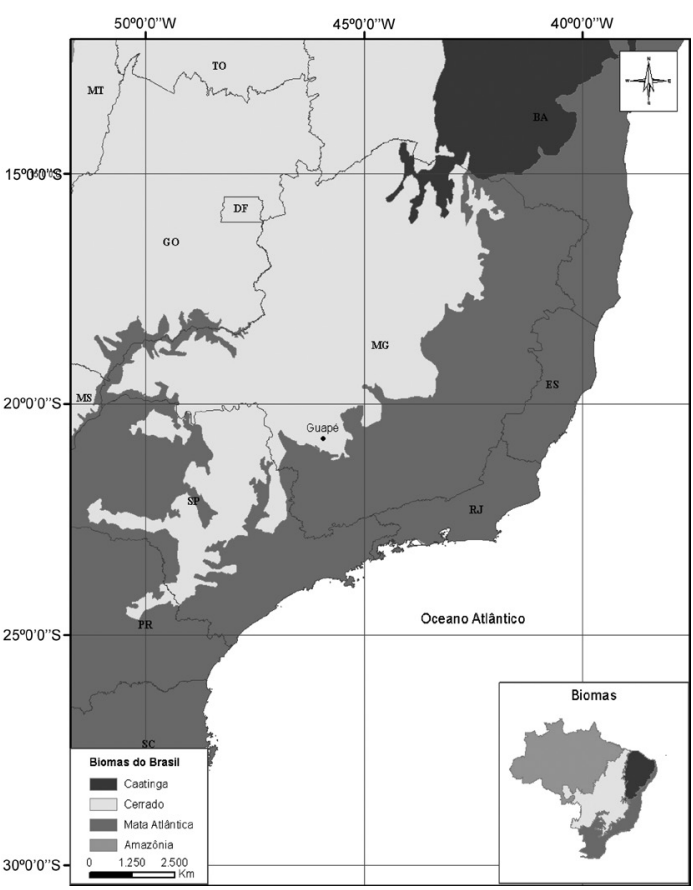

Figura 1 - Mapas com a identificação dos fragmentos e das unidades amostrais inventariadas no município de Guapé em relação aos Domínios Fitogeográficos do estado de Minas Gerais e a caracterização fitofisionômica da região. $\mathrm{CR}=$ cerrado sensu stricto rupestre; CT = cerrado sensu stricto típico.

Figure 1 - Maps with the identification of the fragments and sample units inventoried in the municipality of Guapé regarding to the phytogeographic domains of Minas Gerais and the characterization of the vegetation types in the region. $\mathrm{CR}=$ rupestral cerrado sensu stricto; $\mathrm{CT}=$ typical cerrado sensu stricto.

do Sul de Minas Gerais (Dalanesi et al. 2004), com pastagens, lavouras e florestas estacionais semideciduais sobre solos mais profundos e o cerrado sensu latu e campos naturais nas áreas de solos mais rasos. Embora IBGE (2004) compreendam a área como inclusa no domínio da Mata Atlântica, a região abriga uma das áreas de transição entre os domínios Atlântico e do Cerrado (Fig. 1).

\section{Amostragem da vegetação}

Foram alocadas ao todo 14 parcelas permanentes de $50 \times 30 \mathrm{~m}$, oito no cerrado rupestre e seis no cerrado típico, totalizando 2,1 ha. Todos os indivíduos arbóreos presentes nas parcelas com circunferência de $30 \mathrm{~cm}$ acima do nível do solo $(\mathrm{C} 30) \geq 15,7 \mathrm{~cm}$ (diâmetro de $5 \mathrm{~cm}$ ) foram mensurados com fita métrica, segundo a metodologia proposta por Felfili et al. (2005), e tiveram suas respectivas alturas tomadas. Os indivíduos registrados foram identificados e numerados com placas metálicas dos quais foram coletados ao menos uma exsicata de cada espécie a fim de compor a coleção botânica de referência. Após a herborização do material, estas foram identificadas por comparação e com auxílio de especialistas. Coletas florísticas também foram executadas, onde as espécies arbóreas em estádio fértil presentes fora das parcelas foram coletadas para complementar a listagem de espécies. Toda a coleção botânica foi depositada no Herbário ESAL, da Universidade Federal de Lavras (UFLA). Para classificação das espécies utilizouse o sistema de classificação do Angiosperm Phylogeny Group (APG III 2009). A nomenclatura botânica foi conferida e sinonimizada através da ferramenta digital Plantminer (Carvalho et al. 2010), considerando o banco de dados da Flora do Brasil 2020 (JBRJ 2016).

Amostragem e classificação dos solos

Em cada parcela o solo foi classificado e categorizado segundo o Sistema Brasileiro de Classificação de Solos (EMBRAPA 1999). Em seguida, com auxílio de um trado, uma amostra composta de cinco subamostras de solo superficial - 0 a $20 \mathrm{~cm}$ - foi coletada em cada parcela. As amostras foram enviadas ao Laboratório de Análise de Solos da UFLA para análises químicas e texturais de acordo com o protocolo adotado pela EMBRAPA (1997). As variáveis obtidas foram $\mathrm{pH}$, teores de $\mathrm{P}, \mathrm{K}, \mathrm{Ca}, \mathrm{Mg}, \mathrm{Al}$, P remanescente (P-rem) e matéria orgânica $(\mathrm{MO})$, índice de saturação de bases (V), soma de bases (SB), capacidade de troca de cátions $(\mathrm{T})$ e teores de areia, silte e argila.

\section{Análises dos dados}

Com diferenças de esforço amostral entre as áreas, a diversidade florística local foi analisada por meio de curvas de rarefação por indivíduos (Moro \& Martins 2011) e de distribuição de abundância de espécies (Magurran 1988). A estrutura das comunidades foi comparada através dos descritores fitossociológicos de abundância de indivíduos $(\mathrm{N})$, parcelas de ocorrência $(\mathrm{P})$, densidade absoluta (DA), dominância absoluta (DoA), além do índice de valor de importância - IVI (Moro \& Martins, 2011). Foram também obtidos os gráficos de distribuição de frequências de diâmetro e altura da comunidade, além dos índices de diversidade de Shannon e equabilidade de Pielou (Brower \& Zar 1984). 
Para determinação dos processos de estruturação das comunidades, os atributos edáficos das duas classes pedológicas levantadas foram comparados pelo teste estatístico de MannWhitney a 5\% de confiança (Hart 2001). Ainda com objetivo de identificar tais mecanismos ecológicos, os parâmetros de densidade de indivíduos e área basal por hectare, bem como médias de diâmetro $(\mathrm{C} 30)$ e de alturas dos diferentes subtipos de cerrado sensu stricto também foram submetidos ao mesmo teste estatístico (Hart 2001). Por fim, para correlacionar os parâmetros de solo e a abundância de espécie, utilizou-se a análise de correspondência canônica - CCA (Ter Braak 1987) do software PC-ORD 5.0 (McCune \& Mefford 1999). Para testar a significância dos autovalores da CCA e as correlações espécie-ambiente obtidas foi realizado o teste de permutação de Monte Carlo (Manly 1997). As matrizes de dados utilizadas para as análises e construção das curvas de rarefação e distribuição de abundância de espécies, bem como de distribuições de frequência, parâmetros edáficos e correlações espécie-ambiente encontram-se disponíveis como suplemento do presente artigo no endereço digital: (<http://dx.doi.org/10.6084/ m9.figshare.4213800>).

\section{Resultados}

Florística e estrutura da vegetação

A diversidade alfa do cerrado estudado, considerando ambos os fragmentos e os levantamentos florístico e fitossociológico, foi de 83 espécies, compreendidas em 65 gêneros de 37 famílias botânicas (Tab. 1). As famílias mais ricas foram Fabaceae com 14 espécies, Vochysiaceae e Asteraceae com 6 espécies cada, e Myrtaceae com 5 espécies. Juntas estas famílias foram responsáveis por $37 \%$ da comunidade levantada. Famílias com apenas uma espécie foram responsáveis por $29 \%$ da flora, ou seja, 24 espécies. Os gêneros mais representativos foram Qualea e Eremanthus, com 4 espécies cada, Erythroxylum e Miconia com 3 espécies cada, e outros oito gêneros apresentaram duas espécies cada.

Nas parcelas de cerrado rupestre foram amostrados, ao todo, 916 indivíduos, distribuídos em 47 espécies de 26 famílias botânicas. As famílias mais ricas foram Fabaceae e Vochysiaceae (6 espécies cada), Myrtaceae e Asteraceae (4 espécies), e Annonaceae com 3 espécies. Juntas estas famílias somaram $49 \%$ da riqueza. Por outro lado, 14 foram as famílias que apresentaram somente uma espécie. Os gêneros mais abundantes foram Qualea e Eremanthus com 3 espécies cada, Xylopia, Erythroxylum, Miconia, Myrcia e Vochysia, 2 espécies cada, o restante (26 gêneros), apresentaram somente uma espécie cada. A família mais abundante no cerrado rupestre foi Vochysiaceae com 269 indivíduos mensurados, seguida de Fabaceae (129), Caryocaraceae (126), Myrtaceae (96) e Asteraceae (80). As espécies com índices de valor de importância (IVI) maiores que 10 foram, em ordem decrescente, Caryocar brasiliense Cambess., Qualea dichotoma (Mart.) Warm., Siphoneugena densiflora O.Berg, Qualea multiflora Mart., Eremanthus mattogrossensis Kuntze, Dalbergia miscolobium Benth., Qualea grandiflora Mart., Qualea parviflora Mart.e Miconia albicans (Sw.) Triana (Tab. 1). Na comunidade, a densidade absoluta e área basal foi de $769,16 \mathrm{ind} / \mathrm{ha}$ e $10,91 \mathrm{~m}^{2} /$ ha respectivamente. O índice de diversidade de Shannon encontrado foi de 3,10 nats/ind. enquanto a equabilidade de Pielou foi de 0,79 .

$\mathrm{Na}$ estrutura do cerrado típico, a densidade absoluta e área basal registrada foi de 717,78 ind/ha e $5,20 \mathrm{~m}^{2} /$ ha respectivamente. Foram inventariados 645 indivíduos de 46 espécies arbóreas e 28 famílias botânicas. O valor do índice de diversidade de Shannon foi de 3,19 enquanto a equabilidade de Pielou de 0,83. Os gêneros mais abundantes foram Qualea (3 espécies), Byrsonima e Miconia (2 espécies cada) e outros 37 gêneros, ou seja $84 \%$ da comunidade, foram representados por somente uma espécie cada. A família mais rica no cerrado típico foi Fabaceae com 10 espécies, seguida de Vochysiaceae e Malpighiaceae (3 espécies cada); 16 foram as famílias com apenas uma espécie. A família mais abundante foi Fabaceae com 268 indivíduos, seguida de Malpighiaceae (82) e Annonaceae (53). As espécies mais importantes em IVI (> 10) foram Dalbergia miscolobium Benth., Platimenia reticulada Benth., Stryphnodendron adstringens (Mart.) Coville, Bowdichia virgilioides Kunth, Xylopia aromatica (Lam.) Mart., Erythroxylum suberosum A.St.-Hil., Heteropterys byrsonimifolia A.Juss., Byrsonima coccolobifolia Kunth, Schefflera macrocarpa (Cham. \& Schltdl.) Frodin, Peltophorum dubium (Spreng.) Taub., Miconia ferruginata DC. e Byrsonima verbascifolia (L.) DC. (Tab. 1). 
Tabela 1 - Famílias e espécies arbóreas registradas no levantamento florístico e fitossociológico de duas comunidades de cerrado sensu stricto no município de Guapé, Sul de Minas Gerais. $\mathrm{N}=$ número de indivíduos; $\mathrm{P}=$ número de parcelas de ocorrência; DA = densidade absoluta; DoA = dominância absoluta; VI = Valor de Importância; *Espécies coletadas apenas no levantamento florístico.

Table 1 - Families and tree species recorded in the floristic and phytosociological survey of two cerrado sensu stricto communities in the municipality of Guapé, south of Minas Gerais. $\mathrm{N}=$ number of individuals; $\mathrm{P}=$ number of plots of occurrence; $\mathrm{DA}=$ absolute density; DoA = absolute dominance; VI = Importance Value; * Species collected only in the floristic survey.

\begin{tabular}{|c|c|c|c|c|c|c|c|c|c|c|}
\hline \multirow{2}{*}{ FAMÍLIA / Espécie } & \multicolumn{5}{|c|}{ Cerrado sensu stricto rupestre } & \multicolumn{5}{|c|}{ Cerrado sensu stricto típico } \\
\hline & $\mathbf{N}$ & $\mathbf{P}$ & DA & DoA & VI & $\mathbf{N}$ & $\mathbf{P}$ & DA & DoA & VI \\
\hline \multicolumn{11}{|l|}{ ANACARDIACEAE } \\
\hline \multicolumn{11}{|l|}{ Tapirira guianensis Aubl. * } \\
\hline \multicolumn{11}{|l|}{ ANNONACEAE } \\
\hline Annona coriacea Mart. & 3 & 3 & 2,50 & 0,021 & 2,47 & 2 & 2 & 2,22 & 0,016 & 2,27 \\
\hline Xylopia aromatica (Lam.) Mart. & 8 & 4 & 6,67 & 0,032 & 3,78 & 51 & 5 & 56,67 & 0,245 & 16,70 \\
\hline Xylopia sericea A.St.-Hil. & 4 & 1 & 3,33 & 0,013 & 1,22 & & & & & \\
\hline \multicolumn{11}{|l|}{ APOCYNACEAE } \\
\hline Aspidosperma tomentosum Mart. & 4 & 2 & 3,33 & 0,019 & 1,92 & & & & & \\
\hline \multicolumn{11}{|l|}{ ARALIACEAE } \\
\hline Dendropanax cuneatus (DC.) Decne. \& Planch. & & & & & & 1 & 1 & 1,11 & 0,003 & 1,03 \\
\hline Schefflera macrocarpa (Cham. \& Schltdl.) Frodin & & & & & & 26 & 6 & 28,89 & 0,147 & 11,78 \\
\hline \multicolumn{11}{|l|}{ ASTERACEAE } \\
\hline \multicolumn{11}{|l|}{ Baccharis dracunculifolia DC. * } \\
\hline \multicolumn{11}{|l|}{ Eremanthus elaeagnus (Mart. ex DC.) Sch.Bip. * } \\
\hline Eremanthus erythropappus (DC.) MacLeish & 1 & 1 & 0,83 & 0,003 & 0,79 & & & & & \\
\hline Eremanthus glomerulatus Less. & 1 & 1 & 0,83 & 0,006 & 0,82 & 16 & 3 & 17,78 & 0,080 & 6,48 \\
\hline Eremanthus mattogrossensis Kuntze & 72 & 5 & 60,00 & 0,578 & 17,36 & & & & & \\
\hline Moquiniastrum polymorphum (Less.) G. Sancho & 6 & 1 & 5,00 & 0,023 & 1,55 & 7 & 3 & 7,78 & 0,028 & 4,09 \\
\hline \multicolumn{11}{|l|}{ BIGNONIACEAE } \\
\hline Handroanthus ochraceus (Cham.) Mattos & 4 & 3 & 3,33 & 0,029 & 2,67 & 2 & 2 & 2,22 & 0,032 & 2,57 \\
\hline \multicolumn{11}{|l|}{ Handroanthus serratifolius (Vahl) S.O.Grose * } \\
\hline \multicolumn{11}{|l|}{ Zeyheria montana Mart. * } \\
\hline \multicolumn{11}{|l|}{ BORAGINACEAE } \\
\hline \multicolumn{11}{|l|}{ Cordia trichotoma (Vell.) Arrab. ex Steud. * } \\
\hline \multicolumn{11}{|l|}{ BURSERACEAE } \\
\hline Protium heptaphyllum (Aubl.) Marchand & 5 & 1 & 4,17 & 0,183 & 3,20 & & & & & \\
\hline \multicolumn{11}{|l|}{ CARYOCARACEAE } \\
\hline Caryocar brasiliense Cambess. & 126 & 8 & 105,00 & 2,260 & 43,63 & & & & & \\
\hline \multicolumn{11}{|l|}{ CELASTRACEAE } \\
\hline Plenckia populnea Reissek & 2 & 1 & 1,67 & 0,007 & 0,93 & & & & & \\
\hline \multicolumn{11}{|l|}{ CHRYSOBALANACEAE } \\
\hline Couepia grandiflora (Mart. \& Zucc.) Benth. & 28 & 6 & 23,33 & 0,273 & 9,88 & & & & & \\
\hline Hirtella gracilipes (Hook.f.) Prance & 3 & 1 & 2,50 & 0,014 & 1,12 & & & & & \\
\hline \multicolumn{11}{|l|}{ CLETHRACEAE } \\
\hline \multicolumn{11}{|l|}{ Clethra scabra Pers. * } \\
\hline CLUSIACEAE & & & & & & & & & & \\
\hline
\end{tabular}




\begin{tabular}{|c|c|c|c|c|c|c|c|c|c|c|}
\hline \multirow{2}{*}{ FAMÍLIA / Espécie } & \multicolumn{5}{|c|}{ Cerrado sensu stricto rupestre } & \multicolumn{5}{|c|}{ Cerrado sensu stricto típico } \\
\hline & $\mathbf{N}$ & $\mathbf{P}$ & DA & DoA & VI & $\mathbf{N}$ & $\mathbf{P}$ & DA & DoA & VI \\
\hline Calophyllum brasiliense Cambess. & 2 & 1 & 1,67 & 0,051 & 1,42 & & & & & \\
\hline Kielmeyera lathrophyton Saddi & & & & & & 2 & 1 & 2,22 & 0,015 & 1,43 \\
\hline Kielmeyera speciosa A.St.-Hil. & 6 & 4 & 5,00 & 0,033 & 3,58 & & & & & \\
\hline \multicolumn{11}{|l|}{ COMBRETACEAE } \\
\hline Terminalia glabrescens Mart. & & & & & & 1 & 1 & 1,11 & 0,006 & 1,09 \\
\hline \multicolumn{11}{|l|}{ CONNARACEAE } \\
\hline Connarus suberosus Planch. & 10 & 4 & 8,33 & 0,036 & 4,04 & & & & & \\
\hline \multicolumn{11}{|l|}{ ERYTHROXYLACEAE } \\
\hline \multicolumn{11}{|l|}{ Erythroxylum citrifolium A.St.-Hil. * } \\
\hline Erythroxylum suberosum A.St.-Hil. & 27 & 8 & 22,50 & 0,126 & 9,44 & 45 & 6 & 50,00 & 0,233 & 16,36 \\
\hline Erythroxylum tortuosum Mart. & 1 & 1 & 0,83 & 0,002 & 0,78 & & & & & \\
\hline \multicolumn{11}{|l|}{ FABACEAE } \\
\hline Leptolobium dasycarpum Vogel & 5 & 1 & 4,17 & 0,036 & 1,58 & 3 & 2 & 3,33 & 0,014 & 2,39 \\
\hline Bowdichia virgilioides Kunth & 13 & 4 & 10,83 & 0,138 & 5,49 & 41 & 6 & 45,56 & 0,300 & 17,02 \\
\hline Copaifera langsdorffii Desf. & & & & & & 16 & 1 & 17,78 & 0,106 & 5,32 \\
\hline Dalbergia miscolobium Benth. & 73 & 6 & 60,83 & 0,464 & 16,86 & 75 & 6 & 83,33 & 0,667 & 29,28 \\
\hline \multicolumn{11}{|l|}{ Dalbergia villosa (Benth.) Benth. * } \\
\hline Dimorphandra mollis Benth. & 2 & 2 & 1,67 & 0,039 & 1,93 & 1 & 1 & 1,11 & 0,009 & 1,15 \\
\hline Enterolobium gummiferum (Mart.) J.F.Macbr. & 15 & 3 & 12,50 & 0,148 & 5,17 & 10 & 3 & 11,11 & 0,117 & 6,26 \\
\hline \multicolumn{11}{|l|}{ Hymenaea stigonocarpa Mart. ex Hayne* } \\
\hline \multicolumn{11}{|l|}{ Machaerium hirtum (Vell.)Stellfeld * } \\
\hline \multicolumn{11}{|l|}{ Machaerium villosum Vogel * } \\
\hline Peltophorum dubium (Spreng.) Taub. & & & & & & 27 & 2 & 30,00 & 0,281 & 11,18 \\
\hline Piptadenia gonoacantha (Mart.) J.F.Macbr. & & & & & & 1 & 1 & 1,11 & 0,008 & 1,13 \\
\hline Plathymenia reticulata Benth. & & & & & & 38 & 6 & 42,22 & 0,606 & 22,39 \\
\hline Stryphnodendron adstringens (Mart.) Coville & 21 & 5 & 17,50 & 0,266 & 8,40 & 56 & 6 & 62,22 & 0,459 & 22,37 \\
\hline \multicolumn{11}{|l|}{ LAMIACEAE } \\
\hline Aegiphila verticillata Vell. & 2 & 1 & 1,67 & 0,029 & 1,18 & 5 & 4 & 5,56 & 0,026 & 4,58 \\
\hline Hyptidendron canum (Pohl ex Benth.) Harley & 1 & 1 & 0,83 & 0,003 & 0,78 & 18 & 1 & 20,00 & 0,205 & 7,53 \\
\hline \multicolumn{11}{|l|}{ Vitex megapotamica (Spreng.) Moldenke* } \\
\hline \multicolumn{11}{|l|}{ LYTHRACEAE } \\
\hline Lafoensia vandelliana Cham. \& Schltdl. & 11 & 4 & 9,17 & 0,059 & 4,40 & & & & & \\
\hline \multicolumn{11}{|l|}{ MALPIGHIACEAE } \\
\hline Byrsonima coccolobifolia Kunth & 2 & 1 & 1,67 & 0,007 & 0,94 & 31 & 5 & 34,44 & 0,202 & 12,78 \\
\hline Byrsonima verbascifolia (L.) DC. & & & & & & 19 & 5 & 21,11 & 0,181 & 10,52 \\
\hline Heteropterys byrsonimifolia A.Juss. & 8 & 5 & 6,67 & 0,042 & 4,53 & 32 & 5 & 35,56 & 0,295 & 14,70 \\
\hline \multicolumn{11}{|l|}{ MALVACEAE } \\
\hline Luehea paniculata Mart. \& Zucc. & & & & & & 18 & 2 & 20,00 & 0,178 & 7,83 \\
\hline \multicolumn{11}{|l|}{ MELASTOMATACEAE } \\
\hline Miconia albicans (Sw.) Triana & 49 & 5 & 40,83 & 0,293 & 11,74 & 3 & 2 & 3,33 & 0,013 & 2,36 \\
\hline Miconia ferruginata DC. & 1 & 1 & 0,83 & 0,007 & 0,83 & 22 & 3 & 24,44 & 0,266 & 10,96 \\
\hline \multicolumn{11}{|l|}{ Miconia tristis Spring * } \\
\hline Tibouchina sellowiana Cogn. & & & & & & 17 & 2 & 18,89 & 0,072 & 5,65 \\
\hline
\end{tabular}




\begin{tabular}{|c|c|c|c|c|c|c|c|c|c|c|}
\hline \multirow{2}{*}{ FAMÍLIA / Espécie } & \multicolumn{5}{|c|}{ Cerrado sensu stricto rupestre } & \multicolumn{5}{|c|}{ Cerrado sensu stricto típico } \\
\hline & $\mathbf{N}$ & $\mathbf{P}$ & DA & DoA & VI & $\mathbf{N}$ & $\mathbf{P}$ & DA & DoA & VI \\
\hline \multicolumn{11}{|l|}{ MELIACEAE } \\
\hline Trichilia pallens C.DC. & & & & & & 1 & 1 & 1,11 & 0,006 & 1,09 \\
\hline \multicolumn{11}{|l|}{ MYRISTICACEAE } \\
\hline Virola sebifera Aubl. & 3 & 1 & 2,50 & 0,021 & 1,20 & 3 & 1 & 3,33 & 0,010 & 1,49 \\
\hline \multicolumn{11}{|l|}{ MYRSINACEAE } \\
\hline Myrsine guianensis (Aubl.) Kuntze & 20 & 5 & 16,67 & 0,079 & 6,24 & 13 & 2 & 14,44 & 0,069 & 4,98 \\
\hline \multicolumn{11}{|l|}{ MYRTACEAE } \\
\hline Campomanesia xanthocarpa (Mart.) O.Berg & 6 & 1 & 5,00 & 0,035 & 1,67 & & & & & \\
\hline \multicolumn{11}{|l|}{ Eugenia aurata O.Berg ** } \\
\hline Myrcia guianensis (Aubl.) DC. & 21 & 6 & 17,50 & 0,099 & 7,21 & & & & & \\
\hline Myrcia tomentosa (Aubl.) DC. & 5 & 3 & 4,17 & 0,038 & 2,88 & 1 & 1 & 1,11 & 0,009 & 1,15 \\
\hline Siphoneugena densiflora O.Berg & 64 & 4 & 53,33 & 0,752 & 17,77 & 2 & 2 & 2,22 & 0,007 & 2,09 \\
\hline \multicolumn{11}{|l|}{ NYCTAGINACEAE } \\
\hline Neea theifera Oerst. & & & & & & 6 & 1 & 6,67 & 0,074 & 3,17 \\
\hline \multicolumn{11}{|l|}{ OCHNACEAE } \\
\hline Ouratea castaneifolia (DC.) Engl. & 4 & 3 & 3,33 & 0,063 & 3,05 & 1 & 1 & 1,11 & 0,009 & 1,16 \\
\hline \multicolumn{11}{|l|}{ PERACEAE } \\
\hline Pera glabrata (Schott) Poepp. ex Baill. & 2 & 2 & 1,67 & 0,100 & 2,60 & & & & & \\
\hline \multicolumn{11}{|l|}{ PROTEACEAE } \\
\hline Roupala montana Aubl. & 1 & 1 & 0,83 & 0,005 & 0,81 & 5 & 3 & 5,56 & 0,014 & 3,52 \\
\hline \multicolumn{11}{|l|}{ RUBIACEAE } \\
\hline Faramea latifolia (Cham. \& Schltdl.) DC. & 4 & 1 & 3,33 & 0,039 & 1,50 & & & & & \\
\hline Palicourea rigida Kunth & & & & & & 2 & 1 & 2,22 & 0,006 & 1,26 \\
\hline Rudgea viburnoides (Cham.) Benth. & & & & & & 3 & 3 & 3,33 & 0,006 & 3,07 \\
\hline \multicolumn{11}{|l|}{ SALICACEAE } \\
\hline \multicolumn{11}{|l|}{ Casearia sylvestris Sw. * } \\
\hline \multicolumn{11}{|l|}{ SAPINDACEAE } \\
\hline Cupania vernalis Cambess. & & & & & & 3 & 1 & 3,33 & 0,010 & 1,47 \\
\hline \multicolumn{11}{|l|}{ SIPARUNACEAE } \\
\hline Siparuna reginae (Tul.) A.DC. & & & & & & 2 & 2 & 2,22 & 0,007 & 2,09 \\
\hline \multicolumn{11}{|l|}{ STYRACACEAE } \\
\hline Styrax camporum Pohl & 7 & 3 & 5,83 & 0,060 & 3,34 & 1 & 1 & 1,11 & 0,005 & 1,07 \\
\hline \multicolumn{11}{|l|}{ URTICACEAE } \\
\hline Cecropia pachystachya Trécul & & & & & & 1 & 1 & 1,11 & 0,053 & 1,99 \\
\hline \multicolumn{11}{|l|}{ VOCHYSIACEAE } \\
\hline Qualea dichotoma (Mart.) Warm. & 93 & 5 & 77,50 & 0,898 & 23,16 & 4 & 2 & 4,44 & 0,030 & 2,84 \\
\hline Qualea grandiflora Mart. & 49 & 6 & 40,83 & 0,513 & 14,79 & 3 & 2 & 3,33 & 0,072 & 3,48 \\
\hline Qualea multiflora Mart. & 75 & 8 & 62,50 & 0,388 & 17,52 & 9 & 3 & 10,00 & 0,054 & 4,89 \\
\hline Qualea parviflora Mart. & 42 & 6 & 35,00 & 0,368 & 12,45 & & & & & \\
\hline Vochysia rufa Mart. & 2 & 1 & 1,67 & 0,011 & 0,97 & & & & & \\
\hline Vochysia thyrsoidea Pohl & 8 & 4 & 6,67 & 0,336 & 7,12 & & & & & \\
\hline Total geral & 923 & 8 & 769,17 & 9,090 & 300,00 & 646 & 6 & 717,78 & 5,245 & 300,00 \\
\hline
\end{tabular}


A distribuição diamétrica de ambas as comunidades apresentou forma de "J-reverso" (Fig. 2a) e a estrutura vertical concentrou-se, nas duas áreas, na classe de 2,5 a 4 m (Fig. 2b). De acordo com o teste Mann-Whitney a 5\% de significância $(p>0,05)$, a estrutura dos dois hábitats se diferenciou em relação à área basal e DAP médio (Tab. 2). Na comparação através da rarefação (Fig. 3a), embora o cerrado típico tenha apresentado suave elevação da riqueza em relação ao cerrado rupestre, é possível observar riqueza similar entre as comunidades. A abundância relativa das espécies nos dois cerrados evidenciou um padrão de diversidade com a dominância de poucas espécies sobre as comunidades (Fig. 3b). Apesar disso, apenas Dalbergia miscolobium Benth. é similar nos dois habitats como espécie importante em VI.
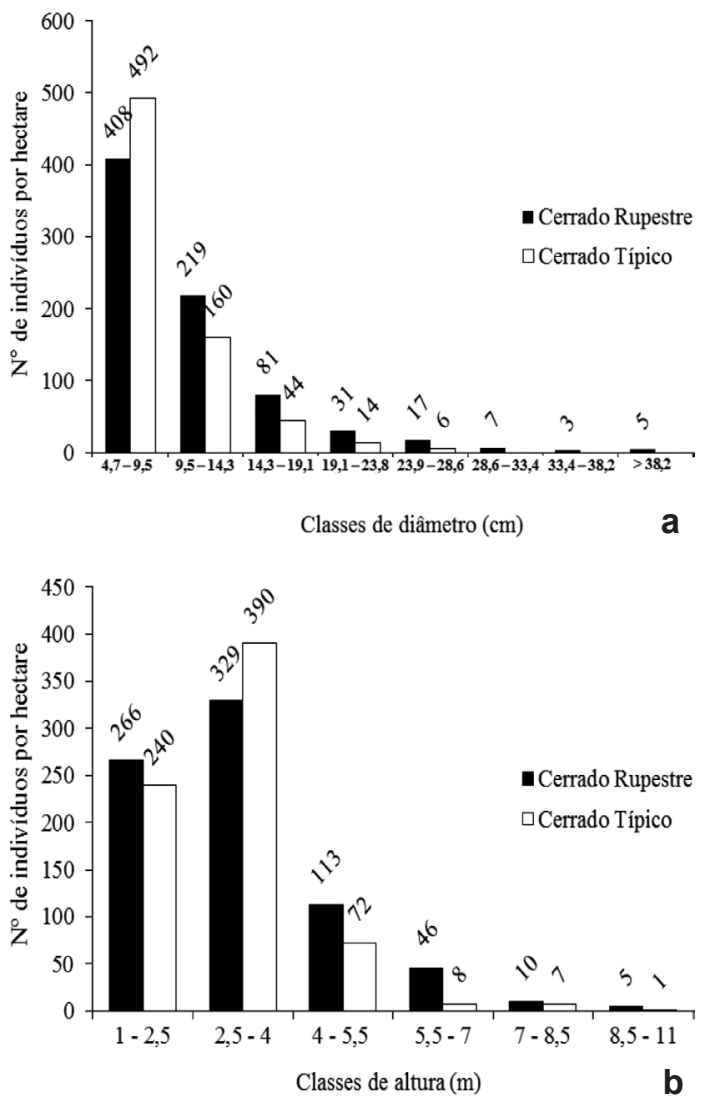

Figura 2 - Distribuição de frequências de classes de diâmetro (a) e altura (b) das comunidades arbóreas de um cerrado rupestre e um cerrado típico no município de Guapé, Sul de Minas Gerais.

Figure 2 - Frequency distribution of diameter classes (a) and height (b) of two tree communities in a rockysoil cerrado and a typical cerrado in the municipality of Guapé, south of Minas Gerais.

\section{Solos}

Os subgrupos de solo identificados foram: neossolo litólico distrófico típico, presente no fragmento de cerrado rupestre, marcado pelo afloramento rochoso da rocha matriz metaquartzítica, e o argissolo vermelho distrófico típico, presente no cerrado típico. Das 14 variáveis de solo comparadas (Tab. 2), 10 diferiram significativamente entre os dois hábitats. $\mathrm{O}$ cerrado rupestre apresentou solo de textura mais arenosa e maiores valores de $\mathrm{P}$, enquanto o cerrado típico
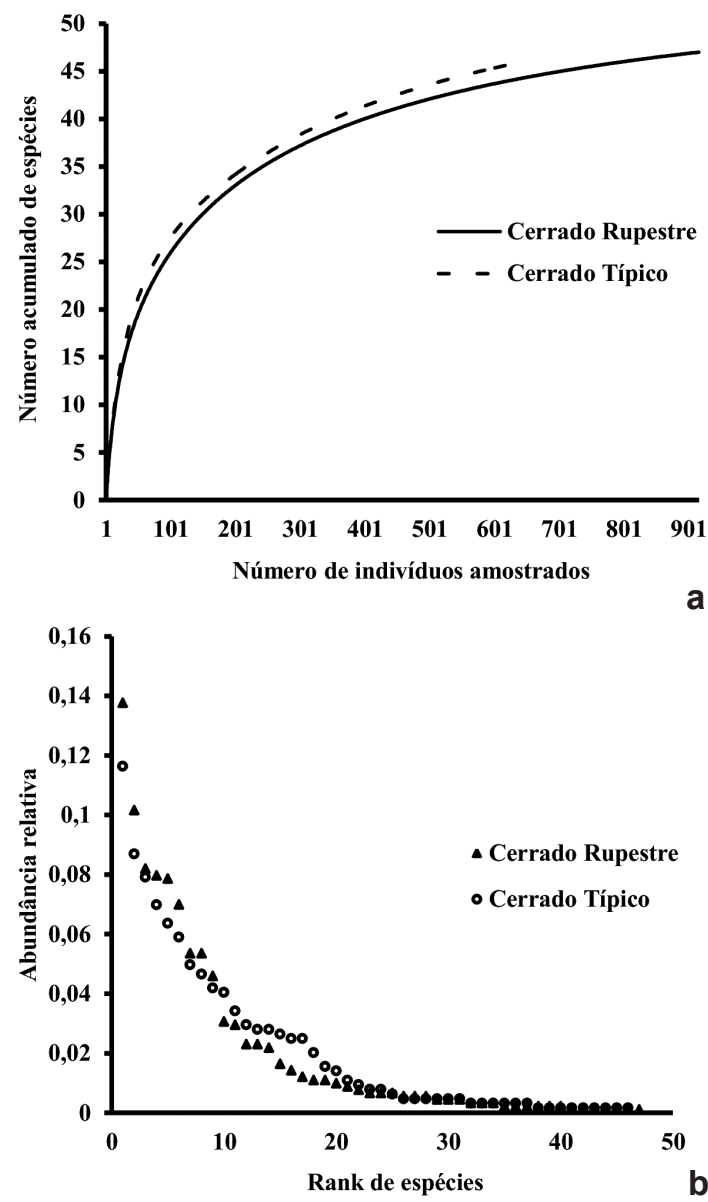

Figura 3 - Curvas de rarefação comparando os levantamentos fitossociológicos arbóreos sobre um cerrado rupestre e um cerrado típico (a); e distribuição de abundância relativa das espécies das duas comunidades arbóreas de cerrado sensu stricto (b) no município de Guapé, Sul de Minas Gerais.

Figure 3 - Rarefaction curves comparing the tree phytosociological surveys on a rockysoil cerrado and a typical cerrado (a); and distribution of relative species abundance in two sensu stricto (b) cerrado communities in the municipality of Guapé, south of Minas Gerais. 
Tabela 2 - Comparação entre variáveis de solo e estruturais da vegetação arbórea registradas em 14 parcelas de cerrado sensu stricto no município de Guapé, Sul de Minas Gerais. Valores são médias \pm desvios padrão das $\mathrm{n}$ amostras de cada classe de solo. (*) Indica diferença significativa segundo teste de Mann-Whitney a $5 \%$ de significância $(p<0,05)$ e $(n . s)$ não significativo.

Table 2 - Comparison between soil and structural variables of trees recorded in 14 sensu stricto cerrado plots in the municipality of Guapé, south of Minas Gerais. Values are means \pm standard deviations of the $n$ samples of each soil class. (*) Indicates significant difference according to Mann-Whitney test at $5 \%$ significance $(\mathrm{p}<0.05)$ and $(\mathrm{n} . \mathrm{s})$ not significant.

\begin{tabular}{lllll}
\hline Atributos & Total (14n) & Neossolo (8n) & Argissolo (6n) & Mann-Whitney (p) \\
\hline $\mathrm{pH}(\mathrm{em} \mathrm{H} \mathrm{O})$ & $4,68 \pm 0,27$ & $4,64 \pm 0,35$ & $4,73 \pm 0,06$ & 0,3935 (n.s) \\
$\mathrm{P}-\mathrm{Mehlich}\left(\mathrm{mg} / \mathrm{dm}^{3}\right)$ & $3,96 \pm 1,95$ & $5,19 \pm 1,72$ & $2,33 \pm 0,16$ & $0,0034^{*}$ \\
$\mathrm{~K}\left(\mathrm{mg} / \mathrm{dm}^{3}\right)$ & $47,21 \pm 30,67$ & $25,38 \pm 6,59$ & $76,33 \pm 17,03$ & $0,0023^{*}$ \\
$\mathrm{Ca}\left(\mathrm{cmol} / \mathrm{dm}^{3}\right)$ & $0,21 \pm 0,32$ & $0,10 \pm 0,00$ & $0,35 \pm 0,23$ & $0,0388^{*}$ \\
$\mathrm{Mg}\left(\mathrm{cmol} / \mathrm{dm}^{3}\right)$ & $0,15 \pm 0,11$ & $0,10 \pm 0,00$ & $0,22 \pm 0,08$ & $0,0116^{*}$ \\
$\mathrm{Al}\left(\mathrm{cmol} / \mathrm{dm}^{3}\right)$ & $1,94 \pm 0,71$ & $1,89 \pm 0,86$ & $2,02 \pm 0,11$ & $0,4371(\mathrm{n} . \mathrm{s})$ \\
$\mathrm{SB}\left(\mathrm{cmol} / \mathrm{dm}^{3}\right)$ & $0,50 \pm 0,49$ & $0,28 \pm 0,05$ & $0,75 \pm 0,31$ & $0,0046^{*}$ \\
$\mathrm{~T}\left(\mathrm{cmol} / \mathrm{dm}^{3}\right)$ & $13,64 \pm 7,64$ & $14,10 \pm 10,19$ & $13,02 \pm 1,81$ & $0,4003(\mathrm{n} . \mathrm{s})$ \\
$\mathrm{V}(\%)$ & $3,94 \pm 3,71$ & $2,45 \pm 1,01$ & $5,92 \pm 1,91$ & $0,0329^{*}$ \\
$\mathrm{MO}(\mathrm{dag} / \mathrm{kg})$ & $3,41 \pm 1,21$ & $3,60 \pm 1,51$ & $3,17 \pm 0,21$ & $0,9482(\mathrm{n} . \mathrm{s})$ \\
$\mathrm{P}-\mathrm{rem}(\mathrm{mg} / \mathrm{L})$ & $34,04 \pm 11,48$ & $40,89 \pm 9,97$ & $24,92 \pm 1,07$ & $0,0136^{*}$ \\
Areia $(\%)$ & $59,43 \pm 20,72$ & $76,00 \pm 5,83$ & $37,33 \pm 10,53$ & $0,0023^{*}$ \\
Silte $(\%)$ & $24,43 \pm 9,35$ & $17,63 \pm 5,68$ & $33,50 \pm 2,60$ & $0,0023^{*}$ \\
Argila $(\%)$ & $16,14 \pm 12,13$ & $6,38 \pm 0,74$ & $29,17 \pm 8,05$ & $0,0015^{*}$ \\
Densidade (ind/ha) & $747,14 \pm 323,57$ & $769,16 \pm 428,57$ & $717,77 \pm 115,71$ & $0,4772(\mathrm{n} . \mathrm{s})$ \\
Área basal (m $/ \mathrm{ha})$ & $15,63 \pm 4,01$ & $10,91 \pm 4,34$ & $4,72 \pm 1,40$ & $0,0238^{*}$ \\
DAP médio (cm) & $10,00 \pm 5,16$ & $10,85 \pm 5,72$ & $8,80 \pm 3,94$ & $0,0081^{*}$ \\
Altura total média (m) & $3,7 \pm 1,29$ & $3,38 \pm 1,44$ & $3,10 \pm 1,00$ & $0,6514(\mathrm{n} . \mathrm{s})$ \\
\hline
\end{tabular}

apresentou maiores percentuais de argila e silte e maiores teores de $\mathrm{K}$ e $\mathrm{Mg}$.

Correlações entre espécies e ambiente Os autovalores da CCA para os três primeiros eixos de ordenação foram 0,585 (eixo 1); 0,390 (eixo 2) e 0,268 (eixo 3), e as correlações de Pearson para esses eixos foram acima de 0,925 . As espécies mais associadas ao cerrado sobre neossolo foram Caryocar brasiliense Cambess., Eremanthus mattogrossensis Kuntze, Lafoensia vandelliana Cham. \& Schltdl, Couepia grandiflora (Mart. \& Zucc.) Benth. e Vochysia thyrsoidea Pohl; enquanto ao cerrado típico sobre argissolo Peltophorum dubium (Spreng.) Taub., Neea theifera Oerst., Luehea paniculata Mart. \& Zucc., Plathymenia reticulata Benth., Byrsonima verbascifolia (L.) DC., Copaifera langsdorffii Desf. e Schefflera macrocarpa (Cham. \& Schltdl.) Frodin.
Os três primeiros eixos da CCA explicaram $34 \%$ da variância dos dados (eixo $1=17,3$; eixo 2 $=9,7$ e eixo $3=7,01)$ e o teste de permutação de Monte Carlo indicou correlação significativa $(P=$ $0,033)$ entre abundância das espécies e variáveis ambientais para os eixos. Com exceção do pH, todas as variáveis edáficas correlacionaram-se significativamente ao primeiro eixo da CCA com valores acima de 0,563 (Tab. 3). Os teores de $\mathrm{P}$ e Areia mantiveram correlações altas e positivas, enquanto o teor de $\mathrm{K}, \mathrm{Mg}$, SB e Argila apresentaram correlações negativas com aquelas (Tab. 3). O primeiro eixo da CCA explicou $51 \%$ da variância dos dados, com a formação ao longo do eixo de dois grupos distintos (Fig. 4): o primeiro formado pelas unidades amostrais sobre neossolo litólico, mais bem drenados, geologicamente mais recentes e com altos teores de P, Areia e P-rem, e o segundo formado pelas parcelas sobre argissolo vermelho, 
Tabela 3 - Correlações intraset da Análise de Correspondência Canônica (CCA) dos dois primeiros eixos de ordenação e matriz de correlações ponderadas para as variáveis dos solos registradas em 14 parcelas de cerrado sensu stricto no município de Guapé, Sul de Minas Gerais. Correlações significativas com valores absolutos maiores que 0,5 ou menores $-0,5$ estão indicadas em negrito.

Table 3 - Intraset correlations of Canonical Correspondence Analysis (CCA) of the first two axes of ordination and correlation matrix weighted to soil variables recorded in 14 sensu stricto cerrado plots in the municipality of Guapé, south of Minas Gerais. Significant correlations with absolute values higher than 0.5 or less than -0.5 are indicated in bold.

\begin{tabular}{|c|c|c|c|c|c|c|c|c|c|}
\hline \multirow{2}{*}{ Parâmetros Edáficos } & \multicolumn{2}{|c|}{ Correlações Intraset } & \multirow{2}{*}{$\mathbf{P}$} & \multirow{2}{*}{$\mathbf{K}$} & \multirow{2}{*}{ P-rem } & \multirow{2}{*}{ Mg } & \multirow{2}{*}{ SB } & \multirow{2}{*}{ pH } & \multirow{2}{*}{ Areia } \\
\hline & Eixo 1 & Eixo 2 & & & & & & & \\
\hline $\mathrm{P}$ & -0.778 & -0.108 & - & & & & & & \\
\hline K & 0.845 & -0.270 & -0.594 & - & & & & & \\
\hline $\mathrm{Mg}$ & -0.612 & 0.528 & -0.446 & 0.836 & -0.301 & - & & & \\
\hline SB & -0.563 & 0.508 & -0.397 & 0.843 & -0.319 & 0.982 & - & & \\
\hline $\mathrm{pH}$ & -0.322 & 0.256 & -0.608 & 0.314 & -0.438 & 0.463 & 0.431 & - & \\
\hline Areia & 0.936 & 0.039 & 0.729 & -0.488 & 0.771 & -0.488 & -0.455 & -0.264 & - \\
\hline Argila & -0.940 & 0.073 & -0.733 & 0.560 & -0.712 & 0.560 & 0.524 & 0.337 & -0.963 \\
\hline
\end{tabular}

menos drenados e de gênese mais antiga que o anterior e, portanto, com menores valores de $\mathrm{pH}$ associado e com altos teores de $\mathrm{K}, \mathrm{Mg}$, SB e Argila.

\section{Discussão}

A riqueza florística levantada - 83 espécies - encontra-se dentro dos padrões observados para os cerrados sensu stricto do sul de Minas Gerais, 70 a 85 espécies (Carvalho \& Martins 1994, 2009). Essa riqueza florística também está próxima daquela levantada por Felfili \& Fagg (2007) em um mosaico de solos rochosos e concressionários no Brasil Central, com 87 espécies, bem como os da província florística sudeste do Brasil (Ratter et al. 2003; Brado \& Durigan 2004; Dantas \& Batalha 2011). Atribui-se este padrão à variação de gradientes da área estudada, onde existe a influência de espécies de fisionomias de entorno (e.g., FESD), destacando-se Clethra scabra Pers., Dalbergia villosa (Benth.) Benth., Erythroxylum citrifolium A.St.-Hil., Luehea paniculata Mart. \& Zucc., Machaerium villosum Vogel, Piptadenia gonoacantha (Mart.) J.F.Macbr., Siparuna reginae (Tul.) A.DC., Tapirira guianensis Aubl., Terminalia glabrescens Mart., e Zanthoxylum rhoifolium Lam. (Oliveira-Filho \& Fontes 2000). Tais transições entre cerrado e florestas semidecíduas dentro da mesma unidade climática ocorrem principalmente devido a variações das propriedades edáficas, como fertilidade, textura e regime hidrológico no solo (Ruggiero et al. 2002; Durigan \& Ratter 2006).

As curvas de rarefação e de distribuição de abundância evidenciaram que os padrões florísticos dos dois cerrados são próximos. Dessa forma, a classificação de Ribeiro e Walter (1998) para os subtipos de Cerrado sensu stricto também se aplicam aos Cerrados sensu stricto do sul de Minas Gerais. Separadamente o cerrado rupestre

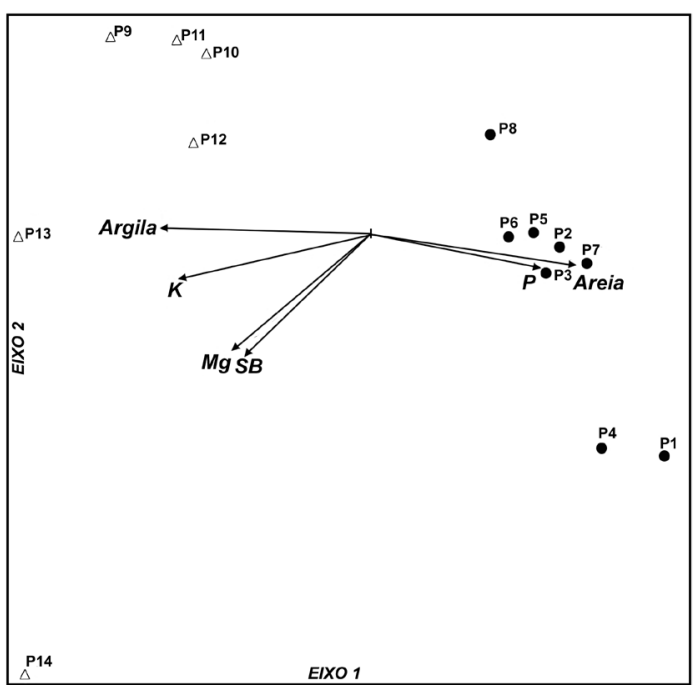

Figura 4 - Análise de Correspondência Canônica (CCA) de duas comunidades de cerrado sobre solos rochosos em Guapé, sudoeste de Minas Gerais. As parcelas representadas por $(\Delta)$ correspondem à área de cerrado típico, enquanto as parcelas representadas por $(\bullet)$ correspondem à área de cerrado rupestre.

Figure 4 - Canonical Correspondence Analysis (CCA) in two cerrado communities on rocky soils in Guapé, southwest of Minas Gerais. The plots represented by $(\Delta)$ correspond to typical cerrado while plots represented by $(\bullet)$ correspond to the cerrado rupestre. 
apresentou riqueza similar a outros trabalhos na fisionomia para a região core do Cerrado (Amaral et al. 2006; Moura et al. 2007, 2010; Pinto et al. 2009). Enquanto o cerrado típico apresentou riqueza próxima aos cerrados transicionais (FESD) da região noroeste de São Paulo (Brando \& Durigan 2004; Dantas \& Batalha 2011).

A distribuição da comunidade em classes de diâmetro encontra-se na forma de "J-reverso", padrão no qual há o recrutamento de indivíduos regenerantes (Felfili 2001). A distribuição em classes de altura apresentou, para os dois fragmentos, maior número de indivíduos na classe de 2,5 a 4,0 m. O padrão de dominância e de valores de importância encontrados para os dois fragmentos foi àquele comum à vegetação dos cerrados, com poucas espécies de grande abundância, aproximadamente 10 espécies representando $50 \%$ de IVI e muitos gêneros e famílias com apenas uma espécie (Felfili et al. 2004). Da mesma forma, as principais famílias botânicas encontradas confirmam o padrão comum para a composição de cerrados sensu stricto no Brasil (Ratter et al. 2003; Bridgewater et al. 2004).

$\mathrm{Na}$ composição de espécies, para os dois ambientes, foram identificadas espécies de ampla distribuição no domínio do Cerrado: Leptolobium dasycarpum Vogel, Annona coriácea Mart., Bowdichia virgilioides Kunth, Byrsonima coccolobifolia Kunth, Caryocar brasiliense Cambess., Dimorphandra mollis Benth., Erythroxylum suberosum A.St.-Hil., Qualea grandiflora Mart., Roupala montana Aubl., Stryphnodendron adstringens (Mart.) Coville e Xylopia aromatic (Lam.) Mart. (Ratter et al. 2003; Bridgewater et al. 2004). Entretanto, coletaramse também espécies raras, como Eremanthus elaeagnus (Mart. Ex DC.) Sch.Bip., Eremanthus mattogrossensis Kuntze e Miconia tristis Spring (Mendonça et al. 2008; Oliveira-Filho 2006).

Valores significativos para os eixos da CCA superiores a 0,5 (eixo 1) apontam para um gradiente curto, com rápida substituição de espécies (Ter Braak 1987). Essa diferença na distribuição da flora no ambiente indica que a explicação para a diversidade beta está correlacionada às características dos solos. Os Neossolos Litólicos são solos rasos, com baixa capacidade de retenção de água, associados a afloramentos de rocha e apresentam elevados teores de minerais primários (Reatto et al. 1998), daí a elevada e positiva correlação "intraset" entre areia e P $(0,729)$. Já o Argissolo apresentou melhor fertilidade, com correlação positiva entre argila e os teores de $\mathrm{K}$ e Mg e também com a SB. Esta classe de solo apresenta profundidade variável, mas um evidente incremento de argila do horizonte B para baixo do perfil (EMBRAPA 1999). Nenhuma relação entre o teor de $\mathrm{MO}$ e argila foi encontrado, embora Goodland \& Ferri (1979) acreditem que a MO seja mais importante que aquela em disponibilizar nutrientes para as plantas em comunidades de cerrado. Pinto et al. (2009) atribuíram a baixa densidade e área basal dos cerrados rupestres à pouca profundidade do solo, alta concentração de alumínio e baixos teores de fertilidade. Contudo, encontrou-se neste trabalho elevada biomassa, comparáveis às outras fisionomias de cerrados sensu stricto (Amaral et al. 2006; Pinto et al. 2009; Moura et al. 2010).

Segundo Assis et al. (2011), o fósforo é um nutriente essencial às plantas, explicando a correlação positiva com elevada área basal. Neri et al. (2012) encontraram correlações positivas entre áreas de elevada área basal e teores de fósforo. Portanto, acredita-se que o que melhor explica e sustenta uma comunidade arbórea de cerrado rupestre sobre neossolos litólicos seja o elevado teor de minerais primários disponíveis. Não obstante, a associação desses minerais com áreas de menor drenagem e maior saturação hídrica resultam na ocorrência de espécies hidrófilas como na parcela três deste levantamento, destacando-se Protium heptaphyllum (Aubl.) Marchand e Calophyllum brasiliense Cambess.

Nas parcelas sobre Argissolo Vermelho, embora este tenha sido classificado como distrófico, a elevação do teor de argila comparado ao Neossolo e o incremento deste no gradiente textural, proporcionou um sítio de maior fertilidade e disponibilidade de água para a vegetação. Assis et al. (2011) sugerem que maiores teores de argila favorecem a retenção de água, pois aumentam a microporosidade do solo, criando condições favoráveis ao estabelecimento de um maior espectro de plantas. Além disso, em Argissolos, ao passo que o teor de argila cresce para o horizonte B (Reatto et al. 1998), a capacidade do solo de retenção de nutrientes aumenta, como observado pela correlação positiva entre K e Mg e SB. Oliveira-Filho \& Ratter (2002), sugerem que a carência de magnésio é um fator limitante ao estabelecimento de vegetações mais densas. Portanto, tais atributos favoreceriam o surgimento de uma vegetação mais densa.

De fato, assim como Dantas \& Batalha (2011) e Assis et al. (2011), acredita-se que é a disponibilidade de água no solo associada à 
fertilidade do substrato, que melhor pode explicar a distribuição das plantas nos cerrados. Isso justifica a ocorrência de espécies de florestas ripárias no domínio do cerrado, como sugere Oliveira-Filho \& Ratter (2002). Da mesma forma, espera-se que a dinâmica sucessional para os cerrado sobre Argissolos seja a mesma descrita para áreas de transição cerrado-floresta sobre solos arenosos da Amazônia (Oliveira-Filho \& Ratter 2002), onde o estádio clímax da vegetação seja o Cerradão. Essa pesquisa indica que em áreas de ecótono cerrado-floresta da província florística sudeste, nos solos com maiores teores de argila, fertilidade e capacidade de retenção de água, o comportamento da vegetação climáxica seja o mesmo observado por Pinheiro \& Durigan (2009), no qual o cerrado sensu stricto conservado tornou-se um Cerradão. Por fim, os cerrados do sul e sudoeste de Minas Gerais comprovam a afirmativa de Bridgewater et al. (2004), na qual os esforços para conservação do Cerrado devem considerar cada província florística em separado.

\section{Material Suplementar}

O presente artigo tem como suplemento digital (disponível em <http://dx.doi.org/10.6084/ m9.figshare.4213800>) duas matrizes de dados brutos, sendo uma dos dados florísticos e fitossociológicos, e outra com os atributos de solo, bem como o mapa apresentado da área de estudo no formato TIF (Tagged Image File Format). Estão também disponíveis quatro tabelas de dados preparados quando da elaboração das representações gráficas apresentadas no artigo.

\section{Agradecimentos}

Ao Assentamento Santo Dias, a oportunidade de realizar este trabalho. A todos os agricultores que participaram das atividades de campo. Ao Programa das Nações Unidas para o Desenvolvimento (PNUD) e ao Instituto Internacional de Educação no Brasil, o financiamento da pesquisa através do Programa Pesco. Ao professor João José G.S. Melo Marques do Departamento de Ciência do Solo da UFLA, a valiosa contribuição nas identificações das classes de solo.

\section{Referências}

Ab' Sáber, A.N. 2003. Os domínios de natureza no Brasil: potencialidades paisagísticas. $3^{\mathrm{a}} \mathrm{ed}$. Ateliê. Editorial, São Paulo. 151p.

APG - Angiosperm Phylogeny Group III. 2009. An update of the Angiosperm Phylogeny Group classification for the orders and families of flowering plants: APG III. Botanical Journal of the Linnaean Society 161: 105-121.

Amaral, A.G.; Pereira, F.F.O. \& Munhoz, C.B.R. 2006. Fitossociologia de uma área de cerrado rupestre na Fazenda Sucupira, Brasília, DF. Cerne 12: 350-359.

Assis, A.C.C.; Coelho, R.M.; Pinheiro, E.S. \& Durigan, G. 2011. Water availability determines physiognomic gradient in an area of low-fertility soils under Cerrado vegetation. Plant Ecology 212: 1135-1147.

Balduino, A.P.C.; Souza, A.L.; Meira-Neto, J.A.A.; Silva, A.F. \& Silva Júnior, M.C. 2005. Fitossociologia e análise comparativa da composição florística do Cerrado da flora de Paraopeba-MG. Revista Árvore 29: 25-34.

Borém, R.A.T. \& Oliveira-filho, A.T. 2002. Fitossociologia do estrato arbóreo em uma toposseqüência alterada de mata Atlântica, no município de Silva Jardim-RJ. Revista Árvore 26: 727-742.

Brasil - Centro Nacional de Ensino e Pesquisas Agronômicas - Comissão de Solos. 1962. Levantamento de reconhecimento dos solos da região sob influência do reservatório de Furnas. Vol. 13. Boletim Técnico do Serviço Nacional de Conservação dos Solos, Rio de Janeiro. 462p.

Brando, P.M. \& Durigan, G. 2004. Changes in cerrado vegetation after disturbance by frost - São Paulo State, Brazil. Plant Ecology 175: 205-215.

Bridgewater, S.; Ratter, J.A. \& Ribeiro, J.F. 2004. Biogeographic patterns, diversity and dominance in the cerrado biome of Brazil. Biodiversity and Conservation 13: 2295-2318.

Brower, J.E. \& Zar, J.H. 1984. Field and laboratory methods for general ecology. W.M.C. Brow, Dubuque. 226p.

Carvalho, D.A. \& Martins, F.R. 1994. Sobre a similaridade de cerrados do sudoeste de Minas Gerais. Cerne 1: 135-145.

Carvalho, D.A. \& Martins, F.M. 2009. Shrub and tree speciescompositionin the cerrado of southwest Minas Gerais. Cerne 15: 142-154.

Carvalho, G.H.; Cianciaruso, M.V. \& Batalha, M.A. 2010. Plantminer: a web tool for checking and gathering plant species taxonomic information. Environmental Modelling \& Software 25: 815-816.

Carvalho, W.A.C.; Oliveira-Filho, A.T.; Fontes, M.A.L. \& Curi, N. 2007. Variação espacial da estrutura da comunidade arbórea de um fragmento de floresta semidecídua em Piedade do Rio Grande, MG. Revista Brasileira de Botânica 30: 321-341.

Couto, W.H.; Anjos, L.H.C.; Toledo, L.O.; Pereira, M.G. \& Queiros, M.M. 2009. Fitossociologia e diversidade florística em área de cerrado sob vários níveis de antropização, Rio Pardo de Minas, MG. Ciência Florestal 19: 351-362.

Dalanesi, P.E.; Oliveira Fiho, A.T. \& Fontes, M.A.L. 2004. Flora e estrutura do componente arbóreo da 
floresta do Parque Ecológico Quedas do Rio Bonito, Lavras, MG, e correlações entre a distribuição das espécies e variáveis ambientais. Acta Botanica Brasilica 18: 737-757.

Dantas, V.L. \& Batalha, M.A. 2011. Vegetation structure: Fine scale relationships with soil in a cerrado site. Flora 206: 341-346.

Durigan, G.; Siqueira, M.F.; Franco, G.A.D.C.; Bridgewater, S. \& Ratter, J.A. 2003. The vegetation of priority areas for cerrado conservation in São Paulo State, Brazil. Edinburgh Journal of Botany 60: 217-241.

Durigan, G. \& Ratter, J.A. 2006. Successional changes in Cerrado and Cerrado/Forest ecotonal vegetation in Western São Paulo State, Brazil, 1962-2000. Edinburgh Journal of Botany 63: 119-130.

Eiten, G. 1994. Vegetação do cerrado. In: Pinto, M.N. (coord.). Cerrado: caracterização, ocupação e perspectivas. $2^{\mathrm{a}}$ ed. UnB/SEMATEC, Brasília. Pp. 9-65.

EMBRAPA - Empresa Brasileira de Pesquisa Agropecuária. 1997. Centro Nacional de Pesquisa de Solos. Manual de métodos de análise de solo. $2^{a}$ ed. Embrapa, Rio de Janeiro. 212p.

EMBRAPA - Empresa Brasileira de Pesquisa Agropecuária. 1999. Centro Nacional de Pesquisa de Solos. Sistema Brasileiro de Classificação de Solos, Brasília. 306p.

Felfili, J.M. 2001. Distribuição de diâmetros de quatro áreas de cerrado sensu stricto na Chapada do Espigão Mestre do São Francisco. In: Felfili, J.M. \& Silva Júnior, M.C. (orgs.). Biogeografia do bioma Cerrado: estudo fitofisionômico na Chapada do Espigão Mestre do São Francisco. Universidade de Brasília, Brasília. Pp. 57-61.

Felfili, J.M.; Silva Júnior, M.C.; Sevilha, A.C.; Fagg, C.W.; Walter, B.M.T.; Nogueira, P.E. \& Rezende, A.V. 2004. Diversity, floristic and structural patterns of cerrado vegetation in Central Brazil. Plant Ecology 175: 37-46.

Felfili, J.M.; Carvalho, F.A. \& Haidar, F.R. 2005. Manual para o monitoramento de parcelas permanentes nos biomas cerrado e pantanal. Universidade de Brasília, Brasília. 60p.

Felfili, J.M. \& Fagg, C.W. 2007. Floristic composition, diversity and structure of the cerrado sensu stricto on rocky soils in northern Goiás and southern Tocantins, Brazil. Revista Brasileira de Botânica 30: 375-385.

Furley, P.A. \& Ratter, J.A. 1988. Soil resouces and communities of the Central Brazilian cerrado and their development. Journal of Biogeography 15: 97-108.

Goodland, R. \& Ferri, M.G. 1979. Ecologia do Cerrado. Universidade de São Paulo, São Paulo. 193p.

Goodland, R. \& Pollard, R. 1973. The Brazilian cerrado vegetation: a fertility gradient. Journal of Ecology 61: 219-224.
Hart, A. 2001. Mann-Whitney test is not just a test of medians: difference in spread can be important. Biometric Journal 323: 391-393.

IBGE - Instituto Brasileiro de Geografia e Estatística. 2004. Mapa de biomas e de vegetação do Brasil. Disponível em $<$ http://www.ibge.gov.br/ $>$.

JBRJ - Jardim Botânico do Rio de Janeiro. 2016. Flora do Brasil 2020 em construção. Disponível em $<$ http://floradobrasil.jbrj.gov.br/>. Acesso em 10 outubro 2016.

Macleish, N.F.F. 1987. Revision of Eremanthus (Compositae: Vernonieae). Annals of the Missouri Botanical Garden 74: 265-290.

Magurran, A.E. 1988. Ecological diversity and its measurement. Princeton University Press, Princeton. 192p.

Manly, B.F.J. 1997. Randomization, bootstrap and Monte Carlo in Biology, $2^{\text {nd }}$ ed. Chapman and Hall, London. 399p.

McCune, B. \& Mefford, M.J. 1999. PC-ORD 4.0: multivariate analysis of ecological data, user guide. MjM Software Design, Oregon.

Mendonça, R.C. et al. 2008. Vascular flora of the Cerrado biome: checklist with 12,356 species. In: Sano, S.M.; Almeida, S.P. \& Ribeiro, J.F. (eds.). Cerrado: ecology and flora. Vol. II. Embrapa Cerrados/Embrapa Informação Tecnológica, Brasília. Pp. 421-1279.

Miranda, I.S.; Absy, M.L. \& Rebêlo, G.H. 2002. Community structure of woody plants of Roraima savannahs, Brazil. Plant Ecology 164: 109-123.

Moura, I.O.; Gomes-Klein, V.L.; Felfili, J.M. \& Ferreira, H.D. 2007. Fitossociologia da comunidade lenhosa de uma área de cerrado rupestre no Parque Estadual dos Pireneus, Pirenópolis, Goiás. Revista de Biologia Neotropical 4: 83-100.

Moura, I.O.; Gomes_Klein, V.L.; Felfili, J.M. \& Ferreira, H.D. 2010. Diversidade e estrutura comunitária de cerrado sensu stricto em afloramentos rochosos no Parque Estadual dos Pireneus, Goiás. Revista Brasileira de Botânica 33: 455-467.

Moreno, M.I.C. \& Schiavini, I. 2001. Relação entre vegetação e solo em um gradiente florestal na Estação ecológica do Panga, Uberlândia, MG. Revista Brasileira de Botânica 24: 537-544.

Moro, M.F. \& Martins, F.R. 2011. Métodos de levantamento do componente arbóreo-arbustivo. In: Felfili, J.M. et al. (ed.). Fitossociologia no Brasil: métodos e estudos de casos. Vol. I. Editora da Universidade Federal de Viçosa, Viçosa. Pp. 174-212.

Neri, A.V.; Schaefer, C.E.G.R.; Silva, A.F.; Souza, A.L.; Ferreira Junior, W.F. \& Meira Neto, J.A.A. 2012. The influence of soils on the floristic composition and community structure of an area of Brazilian cerrado vegetation. Edinbrugh Journal of Botany 69: 1-27. 
Oliveira-Filho, A.T. \& Fontes, M.A.L. 2000. Patterns of floristic differentiation among Atlantic Forest in southeastern Brazil and the influence of climate. Biotropica 32: 793-810.

Oliveira-Filho, A.T. \& Ratter, J.A. 2002. Vegetation physiognomies and woody flora of the Cerrado Biome. In: Oliveira, P.S. \& Marquis, R.J. The Cerrados of Brazil: ecology and natural history of a Neotropical savanna. Columbia University Press, New York. Pp. 91-120.

Oliveira-Filho, A.T. 2006. Catálogo das árvores nativas de Minas Gerais: mapeamento e inventário da flora nativa e dos reflorestamentos de Minas Gerais. Ed. UFLA, Lavras. 423p.

Pinheiro, E.S. \& Durigan, G. 2009. Dinâmica espaçotemporal (1962-2006) das fitofisionomias em unidade de conservação de cerrado no sudeste do Brasil. Revista Brasileira de Botânica 32: 441-454.

Pinto, J.R.R.; Lenza, E. \& Pinto, A.S. 2009. Composição florística e estrutura da vegetação arbustivoarbórea em um cerrado rupestre, Cocalzinho de Goiás, Goiás. Revista Brasileira de Botânica 32: 1-10.

Ratter, J.A.; Bridgewater, S. \& Ribeiro, J.F. 2003. Analysis of floristic composition of Brazilian Cerrado vegetation III: comparison of the woody vegetation of 376 areas. Edinburgh Journal of Botany 60: 57-109.

Ribeiro, J.F. \& Walter, B.M.T. 2008. As principais fitofisionomias do bioma Cerrado. In: Sano, S.M.; Almeida, S.P. \& Ribeiro, J.F. Cerrado: ecologia e flora. EMBRAPA Cerrados, Planaltina. Pp. 151-212.

Reatto, A.; Correia, J.R. \& Spera, S.T. 1998. Solos do Bioma Cerrado: aspectos pedológicos. In: Sano, S.M. \& Almeida, S.P. (eds.). Cerrado: ambiente e flora . Embrapa, Planaltina, Pp.47-86.

Ruggiero, P.G.C.; Batalha, M.A.; Pivello, V.P. \& Meirelles, S.T. 2002. Soil-vegetation relationships in cerrado (Brazilian savana) and semideciduous Forest, South- eastern Brazil. Plant Ecology 160: $1-16$.

Silva, D.M. \& Batalha, M.A. 2008. Soil-vegetation relationships in cerrados under different fire frequencies. Plant Soil 311: 87-96.

Ter Braak, C.J.F. 1987. The analysis of vegetation - environment relationships bay canonical correspondence analysis. Vegetatio 69: 69-77.

Werneck, F.P. 2011. The diversification of eastern South American open vegetation biomes: Historical biogeography and perspectives. Quaternary Science Reviews 30: 1630-1648. 